wishes of people in regard to future treatments and other end of life issues. During its completion, the new form guides the discussion to allow the values and beliefs of a person to be noted, provides relevant information about life prolonging treatments, confirms ongoing palliative care measures and gives reassurance that the dying phase of the illness will not be prolonged.

The cards, the kit and the quill are combined in a new, innovative way to initiate conversations and then to help people express their desires in written form.

Feedback has been positive.

Patients feel that they remain involved in their own care, and that life will continue to have meaning and dignity.

Health professionals state that they now have resources that give structure to their professional relationships, avoid gaps and prevent crises.

\title{
Advance care planning in palliative care practice
}

\section{MAKING DIFFICULT CONVERSATIONS EASIER: THE CARDS, THE KIT AND THE QUILL}

10.1136/bmjspcare-2011-000053.26

E Fairbank South West Healthcare, Australia

The preferences of patients, families and health professionals for preparations for dying have been identified by Steinhauser (JPSM September 2001;22(3)).

Based on this work, CodaAlliance has developed the 'GoWish' card game to help patients communicate their preferences to families and health professionals.

Palliative Care The 'Doing it in Style' Kit, collated by the author, also builds on Steinhauser's work and others, to develop a framework of 10 steps describing effective strategies for preparing for dying. Advance Care Planning features prominently. A Statement of Choices, derived from the Respecting Patient Choices program, Austin Health, Melbourne, Australia has been modified specifically for palliative care use, to document the 